\title{
RELACIONES HOMBRE/NATURALEZA
}





\title{
Raúl Cuadros Contreras \\ Universidad de Ibagué, Colombia \\ raul.cuadros@unibague.edu.co
}

\section{Ontología y epistemología cyborg: representaciones emergentes del vínculo orgánico entre el hombre y la naturaleza ${ }^{1}$}

\author{
Cyborg ontology and cyborg epistemology: emerging \\ representations of the organic link between man and nature
}

Ontologia e epistemologia cyborg: representaçôes emergentes do
vinculo orgânico entre o homem e a natureza

Artículo de investigación científica recibido el 21/09/2010 y aprobado el 02/11/2010

1 Este artículo surge en el marco de mi trabajo de investigación de Maestría y de Doctorado. Ambos trabajos están referidos al estudio de las representaciones de la alteridad en el género de Ciencia Ficción. En el primero se estudian las representaciones de la relación hombre-tecnología entorno a la figura del robot humanoide. En el segundo se indaga las relaciones entre el hombre y muchos otros tipos de seres naturales y artificiales en torno a la figura del monstruo. 



\title{
Resumen
}

El texto presenta la emergencia de una nueva ontología y una nueva epistemología, surgida de transformaciones acaecidas en las representaciones de los objetos de la naturaleza y de la tecnología. Dichas transformaciones implican el paso de consideraciones metafísicas o sustancialistas hacia una perspectiva relacional que los identifica como seres híbridos. Es esa perspectiva relacional la que guía la reconsideración de la identidad humana como el resultado de múltiples vínculos sociales e históricas con otras especies.

Palabras clave: Hombre, Naturaleza, Vínculo Orgánico, Tecnología, Cyborg

\begin{abstract}
This paper addresses the emergence of a new kind of ontology and epistemology, from the transformations occurred in the representations of the objects of nature and technology. These transformations are related with a shift from metaphysical or substantialistic considerations towards a relational perspective that identifies them as hybrid entities. This relational perspective guides the re-consideration of human identity as the result of multiple social and historical links with other species.
\end{abstract}

Key words: Man, Nature, Organic Link, Technology, Cyborg

\section{Resumo}

O texto apresenta a emergência de uma nova ontologia e uma nova epistemologia, surgida de transformaçóes ocorridas nas representaçóes dos objetos da natureza e da tecnologia. Essas transformaçóes envolvem a passagem de consideraçóes metafísicas ou substancialistas para uma perspectiva relacional que os identifica como criaturas híbridas. É essa perspectiva relacional que guia a reconsideração da identidade humana como o resultado de múltiplos vínculos sociais e históricos com outras espécies.

Palavras-chave: Homem, Natureza, Vínculo Orgânico, Tecnologia, Cyborg 



\section{Introducción}

Hasta ahora, en los múltiples esfuerzos por pensar la relación entre el hombre y la naturaleza, prevalecen las consideraciones sustancialistas que se esfuerzan en encontrar las peculiaridades de cada una de las dos identidades. Se quiera o no, se procede al modo aristotélico: buscando la unidad, aquello que permite distinguir inequívocamente a un ser de otros -su sustancia-, de modo que no sea posible la confusión nacida del error de pensar que un ser o un objeto puede ser poseedor de un doble atributo -de ser y no ser a la vez-(Aristóteles, 1967: 947951). Persiste el paradigma de la identidad. Una re-consideración de la relación técnica entre hombre y naturaleza podría reportar nuevos beneficios y ayudar a tomar distancia con respecto a algunas tendencias tanto de la antropología de la técnica como del ecologismo vulgar, que nos devuelven al sustancialismo aristotélico.

La relación hombre naturaleza, es la relación entre un ser vivo artificial y otro ser vivo-artificial, o quizá mejor entre dos seres vivos-artefactuales. El hombre es una creación de sí en su esfuerzo transformador de la naturaleza y, desde su aparición, la naturaleza vino a ser un inmenso ser natural transformado incesantemente, es decir artificial o artefactual. De allí que sea necesario enfocar los problemas atinentes a esta relación desde una perspectiva relacional que indague la constitución de ambos a través de sus mutuas afectaciones. Este cambio de perspectiva supone la asunción de los desplazamientos acaecidos en las maneras de representar tanto a uno como a otra, los cuales tienen que ver con la adopción de la figura del cyborg, que da cuenta de su condición híbrida -natural-artificial. Es necesario señalar que algunos de esos desplazamientos están dando lugar, también, a representaciones problemáticas de lo que entendemos por cultura y de la vieja y naturalizada contraposición naturaleza/cultura.

En lo que sigue se presentarán ejemplos muy diversos que indicarían cómo, en múltiples ámbitos de la investigación científica, se evidencian esos desplazamientos en las formas de representar al hombre y/o a la naturaleza y de concebir las relaciones entre ambos. Todas esas representaciones están atravesadas, ya sea de manera explícita o implícita, por cierta figuración de la tecnología que les proporciona un marco de sentido y que se constituye en una suerte de nuevo paradigma: esta figuración tiene que ver con un modo de pensar emergente que podríamos caracterizar como una ontología y una epistemología cyborg.

\section{La naturaleza como cuerpo inorgánico del hombre en el joven Marx}

Un antecedente temprano de esta perspectiva lo encontramos en las elaboraciones teóricas del joven Marx (1968), que pueden leerse muy a tono con algunos 
de los conceptos y caracterizaciones de Gehlen $(1984,1987,1993)$ pero, también, con las de Gunther Anders (Días, 2010 ). ${ }^{2}$ Marx habla de industria, de trabajo y de intercambio orgánico y se refería a la naturaleza como el cuerpo inorgánico del hombre. A Marx le preocupa el cuerpo y entiende lo humano como algo que tiene que ver, en primer lugar, con la sensibilidad, es por eso que piensa que el hombre no es nada sin la naturaleza, así como cree que no se puede estudiar lo humano si no es como el resultado de una transformación que tiene lugar como resultado del esfuerzo transformador del hombre sobre la naturaleza; pero el hombre mismo es naturaleza y lo que hay de espiritual en él no es otra cosa que corporalidad modificada y sofisticada, y en creciente apertura e indeterminación.

Lo que emerge de la representación de lo humano y de la naturaleza y de la relación específica de lo humano con ella: "la naturaleza como obra suya, como su realidad" (Marx, 1968: 82). Es la imagen de un ser híbrido natural-artificial.

Esta idea temprana es precisada, aún más, en las Tesis sobre Feuerbach (Marx, 2004), en las que Marx formula el sentido de su materialismo en oposición a los materialismos anteriores. Es así como, en la Tesis I empieza diferenciando "cosa" (Gegenstand) de "objeto" (objekt). Todos los materialistas anteriores sólo conciben la realidad como objeto, es decir como algo existente pero nunca como algo producido, como algo que es ante todo el producto de la actividad humana. Ignoran o desestiman que, en el segundo caso, además de los factores objetivos -la materialidad natural- están presentes y son determinantes los factores subjetivos que modifican y han dado forma y una nueva existencia a esa materialidad natural.

Pero también destaca -Tesis V- que éstos, cuando reconocen la actividad sólo la conciben como actividad intelectual o contemplativa, no reconocen la actividad humana ante todo como una actividad sensorial. Y, junto con esto, que la sensibilidad, que los mismos sentidos son producidos por la actividad. Es decir, que al transformar la naturaleza el hombre no sólo produce cosas sino que, también, produce de cierta manera sus propios sentidos, les da nuevas formas y nuevas propiedades, produce sus sentidos como sentidos humanos.

En síntesis, bajo esta perspectiva el mundo, la tierra, es cosa, no objeto, porque es el producto de la interacción entre naturaleza y actividad humana. Pero, al

2 En la medida en que la teoría de la enajenación de Marx tiene como punto de partida la pérdida del objeto por parte del hombre, que pasa a enfrentársele como algo ajeno, así como su concepto del fetichismo según el cual se atribuye a las producciones humanas facultades y potencias que sólo corresponden al propio hombre, ideas como las de: la confusión entre el creador y lo creado -por la vergüenza prometeica-; el desfase entre las necesidades y los productos -o lo que podemos producir y lo que podemos llegar a necesitar o a usar-; y el hecho de no ser ya sujetos de la historia, pues ese lugar pasaría a ser ocupado por los artefactos; pueden ser leídas, perfectamente, en relación con los planteamientos de Marx y hasta ser entendidos sin violencia como una suerte de nueva teoría de la enajenación para la sociedad contemporánea. 
mismo tiempo, el hombre es también cosa, es el producto de la transformación de la naturaleza. Esto último se verifica con mayor fuerza en la medida en que, históricamente, los sentidos humanos son producidos y transformados incesantemente como resultado de esa interacción constante entre hombre y naturaleza, y como resultado de la interacción entre los hombres.

\section{El mundo como alteridad: la teoría Gaia}

La teoría Gaia postula a nuestro planeta como un ser inteligente y sensible, que no es mera objetividad sino un mundo animado. Dicha postulación representa un importante desplazamiento tanto en el plano de las representaciones como en el de los afectos, una importante transformación en el ámbito de la sensibilidad que conlleva la emergencia de una perspectiva nueva tanto en el conocer como en el concebir la tierra y nuestras relaciones con ella:

Digo que Gaia es un sistema fisiológico porque parece tener el objetivo inconsciente de regular el clima y la química de forma que resulten adecuados para la vida [...] Debemos pensar en Gaia como un sistema integral formado por partes animadas e inanimadas. El exuberante crecimiento de los seres vivos, posible gracias al sol, hace a Gaia muy poderosa, pero este caótico y salvaje poder está constreńido por las propias limitaciones de esa entidad que se regula a sí misma en beneficio de la tierra [...] es necesario conocer la verdadera naturaleza de la Tierra e imaginarla como el ser vivo más grande del sistema solar, no como algo inanimado, al modo de esa vergonzosa idea de "la nave espacial Tierra". Hasta que no se produzca este cambio en nuestros corazones y mentes no percibiremos instintivamente que vivimos en un planeta vivo que responderá a los cambios que efectuamos sobre él bien aniquilando los cambios o bien aniquilándonos a nosotros [...] "La Tierra se comporta como un sistema único y autoregulado, formado por componentes físicos, quimicos, biológicos y humanos." (Lovelock, 2007: 38-51) (Los resaltados son nuestros).

La imagen que Lovelock presenta de la tierra es la de un ser heterogéneo, vivo y artificial. Al mismo tiempo, concibe la dinámica de este ser como un asunto relacional porque se trata de un ser animado. Podemos advertir esta misma tendencia o sensibilidad, especie de "estética moral", en la metáfora que Heller y Fehér toman de Heidegger: El hombre es un" pastor del ser”, y su llamado al "cuidado del ser", que ellos traducen como cuidado de la naturaleza, con la cual el pastor actúa "como si" pudiera dialogar:

"El hombre es un pastor del ser" y su llamado al "cuidado del ser", que ellos traducen como cuidado de la naturaleza, con la cual el 
pastor actúa "como si” pudiera dialogar : "La matización ("como si”) no sólo destaca el carácter ficticio del diálogo (el "cuerpo de la naturaleza" es un interlocutor metafórico que nunca puede alcanzar plena autonomía ni llegará jamás a estar realmente articulado), pero también destaca el carácter experimental del acto del pastor. Es una primera exploración de una palabra a la que nos vinculamos, pero de la que aún no nos hemos apropiado. Es en este diálogo cauto y condicional ("como si") y en estos experimentos donde ampliamos nuestro mundo, avanzando siempre con cautela para evitar catástrofes (una posibilidad que nunca preocupó a Fausto) (Heller y Fehér, 1995: 67).

Por supuesto que una actitud como la que reseñamos precisa de un movimiento espiritual previo: es necesario desacralizar nuestra concepción de la vida y abandonar la vieja y dogmática representación que la reduce a algo que se reproduce y corrige los errores de reproducción por el mecanismo de la selección natural, y entenderla como algo mucho más elemental para llegar a pensar a la tierra -o a la parte viva de ella- como un ser vivo. Lovelock declara abiertamente que trabaja con una metáfora, la metáfora de la "tierra viva" para hablar de Gaia. No se refiere a que conciba la tierra como un individuo consciente, pero sí a que se trata de un gran sistema autoregulado que tiende a conseguir equilibrios o estados estables para adaptarse a los cambios climáticos que ponen en peligro las condiciones que hacen posible la existencia de seres vivos en nuestro planeta. Tal descripción es la de un ser sensible que sólo puede existir en función de las múltiples relaciones que entabla con otros seres, relaciones que son, por definición, inestables e históricas.

\section{Cultura en primates no humanos}

La primatología cultural se basa en la hipótesis del aprendizaje social del comportamiento de grupos específicos por parte de primates no humanos. La mayoría de la evidencia proviene de cinco especies: cebus (monos capuchinos), macaca (macacos), gorilla, pongo (orangután) y pan (chimpancés). Dos especies principalmente, el mono japonés (macaca fuscata) y el chimpancé (pan troglodytes), muestran innovación, diseminación, estandarización, durabilidad, difusión y tradición tanto en actividades de subsistencia como de no subsistencia, según lo revelan décadas de estudio longitudinal.

El tema es apasiónate y complejo, pero lo que aquí interesa es el cambio de perspectiva, que desacraliza la noción de cultura desligándola de la "condición humana". "Una manera de determinar la cultura es enfocarse en el proceso" (Kroeber, $1928: 331$ ). 
"Si uno ve que:

1. Un nuevo patrón de comportamiento se inventa o uno existente se modifica.

2. Se transmite ese patrón del innovador a otro.

3. La forma del patrón es consistente dentro y entre los actores, tal vez hasta se puede reconocer un estilo.

4. El patrón persiste en el repertorio de quien lo adquiere mucho después de la ausencia de quien lo mostró.

5. El patrón se extiende a las distintas unidades sociales en una población.

6. El patrón se mantiene a través de las generaciones.

Entonces, uno puede sentirse capaz de reconocer el estatus cultural a ese patrón. La definición adoptada aquí intenta ser lo más incluyente posible: la cultura es considerada como el comportamiento específico de un grupo que es adquirido, al menos en parte, por influencias sociales. Por "grupo" se entiende la unidad de especies típicas, sean una tropa, linaje, subgrupo, etc. (McGrew, 1998: 305).

Semejante postura enfatiza el carácter artefactual de lo humano. Al encontrar lo artefactual en seres no humanos se amplía la propia imagen de lo humano como algo construido, como algo producido, al tiempo que las diferencias entre los humanos y otros animales no se presentan ya como diferencias ontológicas radicales, como diferencias entre esencias:

En conclusión, la cultura primate en sentido amplio parece un rasgo que data por lo menos de nuestro último ancestro antropoide común -con los chimpancés- [...] parafraseando a Gertrude Stein, la cultura es cultura en una variedad de especies, si la cultura se toma como el comportamiento específico de grupo que es por lo menos parcialmente adquirido de influencias sociales. La cultura humana no es igual a la cultura chimpancé ni a la de los monos japoneses. Cada especie es única pero entre más datos se obtienen, las diferencias entre ellas son de grado, no de tipo (McGrew, 1998: 305).

\section{Tecnología humana: interacción social y desarrollo neocortical}

Cierta manera de entender el desarrollo o la evolución corporal y cognitiva se basa en una consideración de la técnica no sólo como la producción social de artefactos, es decir como algo externo, sino como la producción misma del artefacto humano, como técnica humana y social incorporada (Tumbull, 2010). 
Es así como se entiende que llegar al sistema de parentesco australiano fue algo tan necesario como llegar a la construcción de barcos. El desarrollo de formas complejas de conocimiento social, es un prerrequisito para ampliar las relaciones en el espacio y el tiempo. Esta es una mirada que está en consonancia con la "hipótesis del cerebro social" de Robin Dunbar (2008) que sostiene que las sociedades de primates son contratos sociales implícitos establecidos para resolver los problemas ecológicos de la supervivencia y la reproducción de manera más eficaz de lo que podían hacer por su cuenta. David Tumbull postula una explicación de la evolución neocortical en términos de una evolución técnica no sólo exterior como se pensaba tradicionalmente, sino como un desarrollo técnico social, corporal y cognitivo:

Las sociedades de primates trabajan tan eficazmente como lo hacen en este aspecto porque se basan en una vinculación social profunda que es cognitivamente costosa. Por lo tanto, es la demanda de cómputo de la gestión de complejas interacciones la que ha impulsado la evolución neocortical. "Esta concepción de la dinámica de la evolución neocortical humana como social y no simplemente tecnológica encaja bien con, tanto el modelo propuesto por Stanley Ambrose del co-desarrollo del lenguaje, simbolización, un cerebro más grande, y la fabricación de herramientas que se inició en África cerca de 300.000 BP, y con la demanda de Ben Marwick según la cual el lenguaje y la simbolización se desarrollaron con la extensión de redes de intercambio (Tumbull, 2010: 6).

\section{Especies de compañía}

Donna Haraway enfatiza el carácter relacional de lo humano y de la sociedad. Lo que interesa a esta autora en su Manifiesto sobre las especies de compañía (2005) es mostrar que sólo somos en compañía y que no precedemos a las relaciones sociales sino que ellas nos constituyen:

Los seres no preexisten sus relaciones. Las "Prensiones" tienen consecuencias. El mundo es un lazo en movimiento. Los determinismos biológicos y culturales son ambos ejemplos del extravío de lo concreto, el error de, primero, tomar categorías abstractas provisionales y locales como "naturaleza" y "cultura" para el mundo y, segundo, confundir consecuencias poderosas de ser fundaciones preexistentes. No hay sujetos y objetos preconstituídos, no hay fuerzas solitarias, actores únicos o terminaciones finales (2005: 6).

Pero lo novedoso de su planteamiento reside en que concibe esas relaciones sociales como algo que acaece entre múltiples especies y no sólo entre los miem- 
bros de la especie humana, y en que, según esto, no se puede tampoco entender lo humano sino como el resultado en cada momento histórico de la distinta configuración de esas relaciones -configuración que es móvil e inestable. De esta manera, su reflexión contribuye a nuestro esfuerzo por destacar la floración de una ontología y una epistemología cyborg. Haraway afirma: "constitutivamente somos especies en compañía. Nos construimos el uno al otro, en la carne. Significativamente otro con el otro, en diferencias específicas" (2005: 12). Y se propone contar una historia de co-habitación, de co-evolución de sociedades de especies cruzadas, para ello avanza redimensionando a los cyborgs, que pasarían a ser ahora una especie más dentro de ese conjunto de especies en compañía:

\begin{abstract}
Los cyborgs y las especies en compañía brindan cada una lo humano y lo no humano, lo orgánico y lo tecnológico, carbón y silicona, libertad y estructura, historia y mito, riqueza y pobreza, el estado y el sujeto, diversidad y agotamiento, modernidad y postmodernidad, y naturaleza y cultura de maneras insospechadas (Haraway, 2005 : 4).

Especies de compañía es una categoría mucho más grande y heterogénea que animales de compañía, y no sólo porque incluye organismos tales como el arroz, abejas, tulipanes, flora intestinal y todo lo que haga vida para los humanos y viceversa [...] La maquina y lo textual son internas a lo orgánico y viceversa de manera irreversible (Haraway, 2005: 15).
\end{abstract}

\title{
El hombre: una criatura monstruosa
}

Con su insistencia en la alteridad y su apertura hacia una alterización radical, la Ciencia Ficción ayuda a evidenciar las limitaciones del humanismo (Cuadros, 2007 y 2009). Tanto porque enfatiza el carácter relacional de toda identidad y en particular de la identidad humana-la cual sólo es posible en un juego constitutivo de relaciones con otros seres-, así como su condición histórica-artefactual no natural; como porque desplaza de su lugar de centralidad a la especie humana, y a su mundo, y postula la posible condición de sujeto, de persona y de cultural a otros seres distintos de los humanos. Con lo cual, el consabido privilegio de los seres humanos aparece abiertamente cuestionado, y al sugerir con frecuencia la posibilidad de construir nuevas relaciones -no opresivas ni instrumentales-con esos otros, se sugiere también la posibilidad de otro humanismo, moderado y libre de toda angelical ingenuidad.

Como resultado de su confrontación con otras alteridades, el hombre puede ser reinterpretado como una criatura monstruosa. Ante todo como un ser híbrido que se asemejaría más a un centauro, por su constitutiva doble condición animal y cultural. Pero también, en cuanto producto de una construcción constante, en 
cuanto artefacto, como un ser técnico emparentado por esa circunstancia con el robot (Telotte, 1995). La ciencia ficción evidencia esa impureza, deja ver, por contraste, que el hombre no es una esencia completamente distinta de otras, sino que se encuentra emparentado con los animales, con los robots y con los dioses, pero que por su condición híbrida se asemejaría más a un cyborg.

De esta manera la identidad es reducida a una condición impura y problemática, según la cual no tienen sentido insistir en la separación radical o en la discontinuidad ontológica. La especificidad de lo humano no habría que buscarla apelando a la separación, a la mitificación de la identidad, sino profundizando en el estudio de la diferencia - presente en la identidad-, en la indagación del sentido de las diversas relaciones históricas que se han ido tejiendo con otros seres y que dejan su huella en lo humano.

A la idea de Marx de que el hombre -en últimas no tiene esencia-, de que su esencia "es, en su realidad, el conjunto de las relaciones sociales", deberíamos añadir que esas relaciones -que, como él dice, siempre son sociales e históricasincluyen a otros seres distintos de los humanos: a otras especies animales, a el planeta tierra como totalidad y, cada vez más, a todo tipo de artefactos.

\section{Ontología y epistemología cyborg}

Como puede advertirse, la figura del cyborg cubre un espectro mucho más amplio que el acordado a ella tanto por la administración de la ciencia y la tecnología que le dio origen como por el género de ciencia ficción. ${ }^{3}$ Corresponde a toda una ontología y una epistemología, que hunde sus raíces en los cambios en la representación de los objetos de la naturaleza y de la tecnología -los seres vivos y las máquinas. Como sostiene Donna Haraway:

La cultura de la alta tecnología desafía esos dualismos de manera curiosa. No está claro quién hace y quién es hecho en la relación entre el humano y la máquina. No está claro qué es la mente y qué el cuerpo en máquinas que se adentran en prácticas codificadas. En tanto que nos conocemos a nosotras mismas en el discurso formal (por ejemplo, la biología) y en la vida diaria (por ejemplo, la economía casera en el circuito integrado), encontramos que somos cyborgs, híbridos, mosaicos, quimeras. Los organismos biológicos se han convertido en sistemas bióticos, en máquinas de comunicación como las otras. No existe separación ontológica, fundamental en nuestro conocimiento formal de máquina y organismo, de lo técnico y de lo orgánico (Haraway, 1991: 177-178).

3 Cyborg es una palabra inventada por la NASA en 1960 y que significa: ser humano hipotético, constituido con miras a adaptarse a la vida de ambientes no terrestres mediante la sustitución de partes de su cuerpo por órganos artificiales (Haraway, 2005: 4). En la Ciencia Ficción cyborg es todo ser híbrido entre ser orgánico y máquina. 
Bajo esta perspectiva, enriquecida por investigaciones de muy diverso tipo, resulta factible abandonar la imagen separatista de la cultura y la naturaleza, de lo técnico y de lo viviente, que no son más que variantes del dualismo tradicional cuerpo-espíritu o cuerpo mente. ${ }^{4}$

Es en este amplio sentido que deseo sostener la emergencia y la necesidad de una ontología y una epistemología cyborg, las cuales han ido cobrando forma -cuando no-a partir de la irrupción de nuevas metáforas, en las reformulaciones de las nociones y de las categorías que describen a los seres de la naturaleza y a los objetos técnicos y sus relaciones. Pero lo destacable es que esta nueva perspectiva encuentra su modelo en un modo de experiencia signada por la presencia de los objetos técnicos, seres de difícil ontología, que no pueden ser pensados por fuera de sus similitudes y de su estrecha relación con los seres humanos.

\section{Referencias}

Cuadros Contreras, R. (2008). Técnica y alteridad: el robot humanoide, un monstruo problemático y promisorio. En: Criaturas y saberes de lo monstruoso. Buenos Aires: Universidad de Buenos Aires Facultad de Filosofía y Letras.

Cuadros, R. (2008). Perspectivas sobre el humanismo. Ibagué: Universidad de Ibagué.

Días Inserath. C. (2010). “Técnica y singularidad en Gunther Anders y Gilbert Simondon", Revista Iberoamericana de Ciencia, Tecnología y Sociedad. Vol. 5 (14).

Dunbar, R. (2008). "Kinship in Biological Perspective". En: Allen, N.; Callan, H.; Dunbar, R. \& James, W. Early Human Kinship: From Sex to Social Reproduction, edited by Oxford: Blackwell. pp. 131-150.

Gehlen, A. (1984). L'uomo nell era della técnica. Problemi socio-psicologici della civiltá industriale. Milán: SugarCo. . (1987). El hombre. Salamanca: Sígueme. . (1993). Antropología filosófica. Madrid: Paidós.

Haraway, D. (1991). Simios, Cyborgs and Woman: The Reinvention of Nature. New York: Routledge.

$4 \mathrm{Al}$ respecto resulta necesario destacar lo dicho por Simondon: "La oposición que se ha erigido entre la cultura y la técnica, entre el hombre y la máquina, es falsa y sin fundamentos; sólo recubre ignorancia o resentimiento. Enmascara detrás de un humanismo fácil una realidad rica en esfuerzos humanos y en fuerzas naturales, y que constituye en mundo de los objetos técnicos, mediadores entre la naturaleza y el hombre” (2008: 32). 
(2005). The companion species manifesto: Dogs, people, and significant

othereness. Chicago: Prickly paradigm press.

Heidegger, M. (1986). "La pregunta por la técnica", Revista Universidad de Antioquia, No. 205.

Heller, Á. y Fehér, F. (1995). Biopolitica: la modernidad y la liberación del cuerpo, Madrid: Península.

Lorenz, K. (1985). Decadencia de lo humano, Barcelona: Plaza \& Janés.

Lovelock, J. (2007). La venganza de la tierra: la teoría de Gaia y el futuro de la humanidad, Buenos Aires: Planeta.

McGrew, W.C. (1998). "Culture in Nonhuman primates?". Annuel Review of Antropology, Vol 27. pp. 301-328.

Marx, C. (1978). Manuscritos económico filosóficos. México: Fondo de Cultura Económica.

Marx, C. (2004). “Tesis sobre Feuerbach”. La ideología alemana. Buenos Aires: Nuestra América.

Marx, c. (1976). El Capital: Critica de la economía politica, Vol I. México: Fondo de Cultura Económica.

Simondon, g. (2008). El modo de existencia de los objetos técnicos, Buenos Aires: Prometeo.

Telotte, J. P. (1995). Replications: A Robotic History of the Science Fiction Film, Urbana and Chicago: University of Illinois Press.

Tumbull, D. (2010). Other Knowledges, Other Spaces, Other Rationalities: Heterarchy, Complexity and Tension, Norte Chico, Amazonia and Narratives of Prehistory in South America. Ponencia presentada en el Coloquio "Ensamblando a Colombia (I): Naturalezas, Culturas, Tecnologías". Universidad Nacional, agosto 10 al 13. 